\title{
Zeros of quadratic zeta-functions on the critical line
}

\author{
by \\ A. Sankaranarayanan (Bombay) \\ Dedicated to the sixtieth birthday of Professor K. Ramachandra
}

1. Introduction. We follow the usual practice of writing $s=\sigma+i t$. It is a well-known theorem of Hardy that the Riemann zeta-function $\zeta(s)$ has an infinity of zeros on the critical line $\sigma=1 / 2$. In fact, Hardy's proof gives that if $1 / 2+i \gamma_{n}\left(\gamma_{n} \geq 0\right)$ is the $n$th zero of $\zeta(s)$ on $\sigma=1 / 2$ (see [12]), then

$$
\gamma_{n+1}-\gamma_{n} \ll \gamma_{n}^{1 / 4+\varepsilon} \text {. }
$$

The result (1.1) was improved by R. Balasubramanian (see [1]), namely

$$
\gamma_{n+1}-\gamma_{n} \ll \gamma_{n}^{1 / 6+\varepsilon}
$$

In this paper, we consider the zeros of quadratic zeta-functions on the critical line. We begin by explaining the term quadratic zeta-functions. By this, we mean either the Epstein zeta-function associated with a positive definite binary quadratic form or the zeta-function of an ideal class in a quadratic field. One common feature of these things is that each of them has a functional equation of certain type (see $\S 2$, in particular (2.9)).

The main result of this paper is an analogue of (1.1). If $1 / 2+i \gamma_{n}^{*}\left(\gamma_{n}^{*} \geq 0\right)$ is the $n$th zero of any of the quadratic zeta-functions mentioned above, we prove

$$
\gamma_{n+1}^{*}-\gamma_{n}^{*} \ll \gamma_{n}^{* 1 / 2} \log \left(\gamma_{n}^{*}+10\right) .
$$

Some important ground work in this direction has already been built up by H. S. A. Potter and E. C. Titchmarsh [9], E. Hecke [5], K. Chandrasekharan and Raghavan Narasimhan [4], and B. C. Berndt [3]. The main difference between the earlier papers and the present one is that while they argue on the line $\sigma=1+\delta$, we argue on the line $\sigma=1$. For precise results of the earlier authors see (1.8). 
Let

$$
Z(s)=\sum_{m=-\infty}^{\infty} \sum_{n=-\infty}^{\infty} \frac{1}{\left(a m^{2}+b m n+c n^{2}\right)^{s}}=\sum \sum^{\prime} \frac{1}{(\varphi(m, n))^{s}}
$$

in $\sigma>1$. Here $a, b$ and $c$ are real numbers with $a>0, c>0$ and $\Delta=$ $4 a c-b^{2}>0$, so that $\varphi(m, n)$ is a positive definite quadratic form. The dash indicates that the summation is taken over all values of $m$ and $n$ except $m=n=0$. In what follows we take $a, b$ and $c$ are integers with $a>0, c>0$ and $\Delta=4 a c-b^{2}>0$. If $\mathbb{K}$ is a quadratic field and $\mathcal{C}$ is an ideal class in $\mathbb{K}$, then the Dedekind zeta-function of the class $\mathcal{C}$ in $\mathbb{K}$ is defined by the Dirichlet series

$$
\zeta_{\mathbb{K}}(s, \mathcal{C})=\sum_{0 \neq \mathcal{A} \in \mathcal{C}} \frac{1}{(N \mathcal{A})^{s}}
$$

in $\sigma>1$. Here $N \mathcal{A}$ means the norm of the ideal $\mathcal{A} \in \mathcal{C}$. We note that we can write

$$
\zeta_{\mathbb{K}}(s, \mathcal{C})=\sum_{m=1}^{\infty} \frac{a_{m}}{m^{s}} \quad \text { in } \sigma>1
$$

where

$$
a_{m}=\sum_{0 \neq \mathcal{A} \in \mathcal{C}} \frac{1}{(N \mathcal{A})^{s}}
$$

In fact, in [9] H. S. A. Potter and E. C. Titchmarsh proved that

$$
\gamma_{n+1}^{*}-\gamma_{n}^{*} \ll \gamma_{n}^{* 1 / 2+\varepsilon}
$$

for $Z(s)$. In [4], K. Chandrasekharan and Raghavan Narasimhan proved that there are infinitely many zeros on $\sigma=1 / 2$ for $\zeta_{\mathbb{K}}(s, \mathcal{C})$. In [3], Bruce C. Berndt proved (1.8) for $\zeta_{\mathbb{K}}(s, \mathcal{C})$.

R e m a r k. If $\mathcal{C}_{1}, \mathcal{C}_{2}, \ldots, \mathcal{C}_{r}$ are ideal classes in a field $\mathbb{K}=\mathbb{Q}(\sqrt{ \pm d})$, then we can prove an analogue of the inequality (1.3) of the same form for the function $\sum_{j=1}^{r} d_{j} \zeta_{\mathbb{K}}\left(s, \mathcal{C}_{j}\right)$ where the coefficients $d_{j}$ are real constants.

2. Notation and preliminaries. $C_{1}, C_{2}, \ldots, A_{1}, A_{2}, \ldots$ denote positive constants unless it is specified. We write $f(x) \ll g(x)$ to mean $|f(x)|<$ $C_{1} g(x)$ (sometimes, we use the $O$-notation to mean the same). We write $s=\sigma+i t, s_{0}=1+i t$ and $w=u+i v$. Let $\lambda=|d|$ and $\Delta=4 a c-b^{2}>0$. All the constants $C_{1}, C_{2}, \ldots, A_{1}, A_{2}, \ldots$ are effective. The implied constants from $\ll$ and $O$ also are effective. In any fixed strip $\alpha \leq \sigma \leq \beta$, as $t \rightarrow \infty$, we have

$$
\Gamma(\sigma+i t)=t^{\sigma+i t-1 / 2} e^{-\pi t / 2-i t+(i \pi / 2)(\sigma-1 / 2)} \sqrt{2 \pi}(1+O(1 / t)) .
$$


$Z(s)$ satisfies the functional equation (see [6] or [11]):

$$
\left(\frac{\sqrt{\Delta}}{2 \pi}\right)^{s} \Gamma(s) Z(s)=\left(\frac{\sqrt{\Delta}}{2 \pi}\right)^{1-s} \Gamma(1-s) Z(1-s),
$$

and $\zeta_{\mathbb{K}}(s, \mathcal{C})$ satisfies the functional equation (see $[6]$ )

$$
\left(\frac{\sqrt{\lambda}}{2 \pi}\right)^{s} \Gamma(s) \zeta_{\mathbb{K}}(s, \mathcal{C})=\left(\frac{\sqrt{\lambda}}{2 \pi}\right)^{1-s} \Gamma(1-s) \zeta_{\mathbb{K}}(1-s, \mathcal{C}) \quad \text { if } d<0
$$

and

$$
\begin{aligned}
\left(\frac{\sqrt{\lambda}}{\pi}\right)^{s} \Gamma^{2}\left(\frac{s}{2}\right) & \zeta_{\mathbb{K}}(s, \mathcal{C}) \\
& =\left(\frac{\sqrt{\lambda}}{\pi}\right)^{1-s} \Gamma^{2}\left(\frac{1-s}{2}\right) \zeta_{\mathbb{K}}(1-s, \mathcal{C}) \quad \text { if } d>0 .
\end{aligned}
$$

If we write

$$
\begin{aligned}
& Z(s)=\chi_{1}(s) Z(1-s), \\
& \zeta_{\mathbb{K}}(s, \mathcal{C})=\chi_{2}(s) \zeta_{\mathbb{K}}(1-s, \mathcal{C}) \quad \text { if } d<0 \text {, } \\
& \zeta_{\mathbb{K}}(s, \mathcal{C})=\chi_{3}(s) \zeta_{\mathbb{K}}(1-s, \mathcal{C}) \quad \text { if } d>0 \text {, }
\end{aligned}
$$

from (2.2)-(2.4), we get $\left|\chi_{j}(1 / 2+i t)\right|=1$ for $j=1,2,3$, since $\chi_{j}(s)$ is real for real $s$. Since $\Gamma(s)$ has no zeros and only real poles, the function $\left\{\chi_{j}(s)\right\}^{-1}$ has a square root $\left(\chi_{j}(s)\right)^{-1 / 2}$ in the simply connected region $t \geq t_{0}\left(t_{0}\right.$ large enough). We define

$$
W_{j}(t)=f_{j}(1 / 2+i t), \quad f_{j}(s)=G(s) / \sqrt{\chi_{j}(s)}
$$

where

$$
G(s)= \begin{cases}Z(s) & \text { if } j=1 \text { (defined by }(2.2) \text { ), } \\ \zeta_{\mathbb{K}}(s, \mathcal{C}) ; d<0 & \text { if } j=2 \text { (defined by }(2.3) \text { ), } \\ \zeta_{\mathbb{K}}(s, \mathcal{C}) ; d>0 & \text { if } j=3 \text { (defined by }(2.4) \text { ). }\end{cases}
$$

We note that $f_{j}(s)=f_{j}(1-s)$ for $j=1,2,3$ and hence $W_{j}(t)$ is real for real $t$. The zeros of $Z(s), \zeta_{\mathbb{K}}(s, \mathcal{C})$ (with $\left.\mathbb{K}=\mathbb{Q}(\sqrt{d}), d<0\right), \zeta_{\mathbb{K}}(s, \mathcal{C})$ (with $\mathbb{K}=\mathbb{Q}(\sqrt{d}), d>0)$ on $\sigma=1 / 2$ respectively correspond to the real zeros of $W_{1}(t), W_{2}(t)$ and $W_{3}(t)$. From (2.1)-(2.4), it follows that, for $1 / 2 \leq \sigma \leq 1$, we have

$$
\left(\chi_{j}(s)\right)^{-1 / 2}=\left(\frac{M_{j}}{2 \pi}\right)^{\sigma-1 / 2} t^{\sigma-1 / 2}\left(\frac{t M_{j}}{2 \pi e}\right)^{i t} e^{(i \pi / 2)(\sigma-1 / 2)}(1+O(1 / t))
$$

for $j=1,2,3$ where $M_{1}=\sqrt{\Delta}, M_{2}=M_{3}=\sqrt{\lambda}$. 
Let $T \geq T_{0}$ ( $T_{0}$ is a large positive constant) and let $T \leq T^{\prime} \leq 2 T$. For $\mu>0$, we define

$$
J=\int_{T}^{T^{\prime}} t^{\mu}\left(\frac{t}{e \xi}\right)^{i t} d t, \quad \xi>0 .
$$

\section{Some lemmas}

Lemma 3.1. For $\mu>0$, we have

$$
\begin{gathered}
J=O\left(T^{\mu} / \log (T / \xi)\right) \quad \text { if } \xi<T, \\
J=O\left(T^{\mu} / \log \left(\xi / T^{\prime}\right)\right) \quad \text { if } \xi>T^{\prime}, \\
J=(2 \pi)^{1 / 2} \xi^{\mu+1 / 2} e^{i \pi / 4-\xi}+O\left(T^{\mu+2 / 5}\right)+O\left(T^{\mu} / \log (\xi / T)\right) \\
+O\left(T^{\mu} / \log \left(T^{\prime} / \xi\right)\right) \quad \text { if } T<\xi<T^{\prime}
\end{gathered}
$$

and

$$
J=O\left(T^{\mu+1 / 2}\right) \quad \text { for all } \xi>C_{2} .
$$

Proof. (3.1.1) and (3.1.2) follow by using the first derivative test. (3.1.3) follows by the saddle point method and (3.1.4) follows on using the second derivative test. For example see [9].

R e m a r k. For a more general version of Lemma 3.1, we refer to [4]. The estimate (3.1.4) with $\mu>0$ is due to Landau.

LEMMA 3.2. If $R(x)$ is the number of lattice points inside or on the ellipse

$$
a_{1}\left(u-u_{0}\right)^{2}+b_{1}\left(u-u_{0}\right)\left(v-v_{0}\right)+c_{1}\left(v-v_{0}\right)^{2}=x
$$

where $a_{1}, b_{1}, c_{1}, u_{0}, v_{0}$ are fixed, then

$$
R(x)=2 \pi\left(4 a_{1} c_{1}-b_{1}^{2}\right)^{-1 / 2} x+O\left(x^{1 / 2}\right) .
$$

Pro of. See for example VII. Teil, Kap. 7 of [7]. It is given for a circle and it is applicable for the ellipse also.

LEMma 3.3. If $l(j)$ denotes the number of representations of $j$ as $j=$ $a m^{2}+b m n+c n^{2}$, then

(i) $\sum_{j \leq x} l(j)=C_{3} x+O\left(x^{1 / 2}\right)$,

(ii) $\sum_{m \leq x} a_{m}=C_{4} x+O\left(x^{1 / 2}\right)$,

where $a_{m}$ is as defined in (1.7).

Proof. (i) and (ii) follow from Lemma 3.2.

Lemma 3.4. For $t \geq C_{5}$, we have

(i) $Z(1+i t) \ll \log t$,

(ii) $\zeta_{\mathbb{K}}(1+i t, \mathcal{C}) \ll \log t$. 
Proof. First we note that $Z(s)$ is of finite order (see [8]). Hence

$$
Z(\sigma+i t) \ll t^{C_{6}},
$$

where $C_{6} \geq 5$, uniformly for $1 / 2 \leq \sigma \leq 3$. By Mellin's inverse transform, we have

$$
\begin{aligned}
& \sum_{\varphi} \frac{e^{-\varphi / X_{1}}}{\varphi^{s_{0}}}=\frac{1}{2 \pi i} \int_{2-i \infty}^{2+i \infty} Z\left(s_{0}+w\right) \Gamma(w) X_{1}^{w} d w \\
& =\frac{1}{2 \pi i} \int_{\substack{u=2 \\
|v| \leq(\log t)^{2}}} Z\left(s_{0}+w\right) \Gamma(w) X_{1}^{w} d w+O\left(X_{1}^{2} e^{-C_{7}(\log t)^{2}}\right) .
\end{aligned}
$$

Note that $s_{0}=1+i t$. In the integral of the right hand side of (3.4.2), we move the line of integration to $u=-1 / 2$. The pole $w=0$ contributes $Z\left(s_{0}\right)$. The horizontal portions contribute an error which is $O\left(t^{C_{6}} e^{-C_{8}(\log t)^{2}} X_{1}^{2}\right)$. We notice that

$$
\frac{1}{2 \pi i} \int_{\substack{u=-1 / 2 \\|v| \leq(\log t)^{2}}} Z\left(s_{0}+w\right) \Gamma(w) X_{1}^{w} d w=O\left(t^{C_{6}} X_{1}^{-1 / 2}\right) .
$$

Hence, from (3.4.2) we obtain

$$
\begin{gathered}
\sum_{\varphi} \frac{e^{-\varphi / X_{1}}}{\varphi^{s_{0}}}=Z\left(s_{0}\right)+O\left(t^{C_{6}} X_{1}^{-1 / 2}\right)+O\left(t^{C_{6}} X_{1}^{2} e^{-C_{9}(\log t)^{2}}\right), \\
\sum_{\varphi} \frac{e^{-\varphi / X_{1}}}{\varphi^{s_{0}}}=O\left(\sum_{\varphi \leq X_{1}} \frac{1}{\varphi}\right)+O\left(X_{1} \sum_{\varphi>X_{1}} \frac{1}{\varphi^{2}}\right) .
\end{gathered}
$$

Since $\varphi$ is a positive definite quadratic form, from Lemma 3.3(i) we obtain

$$
\sum_{\varphi \leq X_{1}} \frac{1}{\varphi}=\sum_{n \leq X_{1}} \frac{l(n)}{n} \ll \log X_{1},
$$

where $l(n)$ is the number of representations of $n$ as $n=\varphi(x, y)$. Also,

$$
X_{1} \sum_{\varphi>X_{1}} \frac{1}{\varphi^{2}}=X_{1} \sum_{n>X_{1}} \frac{l(n)}{n^{2}} \ll 1 .
$$

We choose $X_{1}=t^{2 C_{6}}$. Hence (i) follows from (3.4.4)-(3.4.7). The proof of (ii) follows in a similar way.

Lemma 3.5. For $t \geq 10$, we have

(i) $Z(\sigma+i t) \ll t^{1-\sigma} \log t$,

(ii) $\zeta_{\mathbb{K}}(\sigma+i t, \mathcal{C}) \ll t^{1-\sigma} \log t$

uniformly for $0 \leq \sigma \leq 1$. 
Pr o o f. (i) From Lemma 3.3, we have

$$
Z(1+i t) \ll \log t
$$

From the functional equation (2.2), and (3.5.1), we get

$$
Z(i t) \ll t \log t .
$$

We apply the maximum-modulus principle to the function

$$
F(w)=Z(w) e^{(w-s)^{2}} X_{2}^{w-s}
$$

in the rectangle defined by the line segments joining the points $i\left(t-(\log t)^{2}\right)$, $1+i\left(t-(\log t)^{2}\right), 1+i\left(t+(\log t)^{2}\right), i\left(t+(\log t)^{2}\right)$ and $i\left(t-(\log t)^{2}\right)$. Now,

$$
|Z(s)| \ll V_{1}+V_{2}+H_{1}+H_{2},
$$

where $V_{1}, V_{2}$ are the contributions from the vertical lines and $H_{1}, H_{2}$ are the contributions from the horizontal lines. We notice that $H_{1} \ll 1$ and $H_{2} \ll 1$. From (3.5.1)-(3.5.3), we obtain

$$
|Z(s)| \ll t(\log t) X_{2}^{-\sigma}+(\log t) X_{2}^{1-\sigma}+1 .
$$

Choosing $X_{2}=t$, we obtain (i). (ii) follows in a similar way.

Lemma 3.6. Let $T \leq t \leq 2 T$ and $X_{3}=\sqrt{\Delta} T^{4}$. We have

$$
Z\left(s_{0}\right)=\sum_{\varphi} \frac{e^{-\varphi / X_{3}}}{\varphi^{s_{0}}}+O\left(T^{-3 / 2}(\log T)^{3}\right),
$$

where $s_{0}=1+i t$.

Proof. As we did in Lemma 3.4, we obtain

$$
\begin{aligned}
\sum_{\varphi} \frac{e^{-\varphi / X_{3}}}{\varphi^{s_{0}}}= & \frac{1}{2 \pi i} \int_{\substack{u=-1 / 2 \\
|v| \leq(\log T)^{2}}} Z\left(s_{0}+w\right) \Gamma(w) X_{3}^{w} d w+Z\left(s_{0}\right) \\
& +O\left(X_{3}^{2} e^{-C_{10}(\log T)^{2}}\right)+O\left(T^{1 / 2}(\log T)^{5} e^{-C_{11}(\log T)^{2}} X_{3}^{2}\right) \\
= & Z\left(s_{0}\right)+O\left(T^{1 / 2}(\log T)^{3} X_{3}^{-1 / 2}\right)+O\left(X_{3}^{2} e^{-C_{10}(\log T)^{2}}\right) \\
& +O\left(T^{1 / 2}(\log T)^{5} e^{-C_{11}(\log T)^{2}} X_{3}^{2}\right) .
\end{aligned}
$$

From our choice of $X_{3}=\sqrt{\Delta} T^{4}$, the lemma follows.

Lemma 3.7. Let $T \leq t \leq 2 T$ and $X_{4}=\sqrt{\lambda} T^{4}$. We have

$$
\zeta_{\mathbb{K}}\left(s_{0}, \mathcal{C}\right)=\sum_{m=1}^{\infty} \frac{a_{m}}{m^{s_{0}}} e^{-m / X_{4}}+O\left(T^{-3 / 2}(\log T)^{3}\right) .
$$

Pr o of. This follows in a similar way to Lemma 3.6. 
LEMma 3.8. If $\alpha_{1}$ is irrational, then

$$
\sum_{n=N}^{N^{\prime}} e^{2 \pi i\left(\alpha_{1} n^{2}+\beta_{1} n\right)}=o\left(N^{\prime}-N\right)
$$

as $N^{\prime}-N$ tends to infinity, uniformly with respect to $\beta_{1}$ and $N$.

Proof. See for example [8].

LEMMA 3.9. For every irrational $x$ and $H=H(T) \leq T$ such that $H / \sqrt{T}$ tends to infinity with $T$ we have

$$
\sum_{T \leq m \leq T+H} a_{m} e^{2 \pi i m x}=o(H),
$$

where $a_{m}$ is as defined in (1.7).

Proof. Let

$$
S(T, T+H)=\sum_{T \leq m \leq T+H} a_{m} e^{2 \pi i m x} .
$$

For a given ideal class $\mathcal{C}$ and a non-zero integral ideal $\mathcal{A} \in \mathcal{C}$, we choose a non-zero integral ideal $\mathcal{B} \in \mathcal{C}^{-1}$ such that $\mathcal{A B}=(\alpha)$ for $\alpha \in \mathcal{B}$. We note that $(1, \omega=(d+\sqrt{d}) / 2)$ is a base of the ring of integers of $\mathbb{K}=\mathbb{Q}(\sqrt{d})$. We denote by $\omega^{\prime}=(d-\sqrt{d}) / 2$ the conjugate of $\omega$.

Case (i): $\mathbb{K}=\mathbb{Q}(\sqrt{d})$ with $d>0$. Let $\alpha_{0}$ be a generator of $(\alpha)$ and let $\alpha^{\prime}$ be the conjugate of $\alpha$. We have $\alpha= \pm \alpha_{0} \eta^{r}$ where $\eta(>1)$ is the fundamental unit and $\eta \eta^{\prime}= \pm 1$. ( $\eta^{\prime}$ is the conjugate of $\eta$.) Now, by letting $L=\left|\alpha_{0} / \alpha_{0}^{\prime}\right|$, we find that

$$
\left|\alpha / \alpha^{\prime}\right|=L \eta^{2 r} \text {. }
$$

We choose $r$ to be the least integer such that $L \eta^{2 r} \geq 1$. Hence, we get

$$
1 \leq\left|\alpha / \alpha^{\prime}\right|<\eta^{2} \text {. }
$$

We can write $\alpha=k+l \omega$ with $k>0$. We notice that for a given non-zero integral ideal $\mathcal{A} \in \mathcal{C}$, there exists one and only one $\alpha=k+l \omega$ with $k>0$ such that $\mathcal{A B}=(\alpha)$ and satisfying the condition (3.9.2). For, if there are two, say $\alpha_{11}=k_{1}+l_{1} \omega$ and $\alpha_{12}= \pm\left(k_{2}+l_{2} \omega\right) \eta^{r}$ with $r \geq 1$, then

$$
\eta^{2}>\left|\frac{\alpha_{11}}{\alpha_{11}^{\prime}}\right|=\left|\frac{\alpha_{12}}{\alpha_{12}^{\prime}}\right| \eta^{2 r} \geq \eta^{2 r},
$$

which is a contradiction. If, in (3.9.2), $k=0$ for $\alpha$ then $\alpha=l \omega$ is unique if we specify that $l>0$, for otherwise $\left(l_{1} \omega\right)$ and $\left(l_{2} \omega\right)$ are different ideals. Now,

$$
m=N \mathcal{A}=\left|(k+l \omega)\left(k+l \omega^{\prime}\right)\right|(N \mathcal{B})^{-1}=|P(k, l)|(N \mathcal{B})^{-1},
$$

where

$$
P(k, l)=(k+l \omega)\left(k+l \omega^{\prime}\right)=k^{2}+a_{2} k l+b_{2} l^{2}
$$


with $4 b_{2}-a_{2}^{2}<0$. From (3.9.2), we have

$$
1 \leq \frac{|k+l \omega|}{\left|k+l \omega^{\prime}\right|}<\eta^{2}
$$

and hence,

$$
\left|k+l \omega^{\prime}\right|^{2} \leq|P(k, l)|<\eta^{2}\left|k+l \omega^{\prime}\right|^{2}
$$

and

$$
|P(k, l)| \leq|k+l \omega|^{2}<\eta^{2}|P(k, l)| .
$$

From (3.9.3), we obtain

$$
T(N \mathcal{B}) \leq|P(k, l)| \leq(T+H)(N \mathcal{B}) .
$$

From (3.9.6)-(3.9.8), we get

$$
|k+l \omega| \leq \eta \sqrt{2(N \mathcal{B}) T} \quad \text { and } \quad\left|k+l \omega^{\prime}\right| \leq \sqrt{2(N \mathcal{B}) T} .
$$

Subtracting one from the other of (3.9.9), we get first $|l| \leq C_{12} \sqrt{T}$ and using this we obtain $k \leq C_{13} \sqrt{T}$. For a given $k>0$, from the inequality (3.9.5), we get two intervals (say) $J_{1}$ and $J_{2}$ for $l$. From (3.9.4) and (3.9.8), we get

$$
4 b_{2}(N \mathcal{B}) T \leq\left|4 b_{2} k^{2}+4 b_{2} a_{2} k l+4 b_{2}^{2} l^{2}\right| \leq 4 b_{2}(N \mathcal{B})(T+H)
$$

i.e.,

$$
C_{14} T \leq\left|\left(2 b_{2} l+a_{2} k\right)^{2}+\left(4 b_{2}-a_{2}^{2}\right) k^{2}\right| \leq C_{14}(T+H) .
$$

The inequality (3.9.10) leads to four intervals (say) $J_{3}, J_{4}, J_{5}$ and $J_{6}$ and we notice that

$$
\text { length of } J_{r} \ll H / \sqrt{T} \text { for } r=3,4,5,6 \text {. }
$$

We define the set $S(k)$ for a fixed $k \geq 0$ to be

$$
S(k)=\left\{(k, l) \mid l \in\left(\bigcup_{r=3}^{6} J_{r}\right) \cap\left(J_{1} \cup J_{2}\right)\right\} .
$$

From (3.9.1), we have

$S(T, T+H)=\frac{1}{2}\left\{\sum_{k} \sum_{\substack{l \\ P(k, l) \equiv 0(N \mathcal{B})}}^{*} e^{2 \pi i|P(k, l)| x(N \mathcal{B})^{-1}}\right\}$

(where $*$ indicates that $l$ runs over $S(k)$ for fixed $k \geq 0$ )

$$
=\frac{1}{2}\left\{\sum_{k} \sum_{l}^{*}\left(\frac{1}{N \mathcal{B}} \sum_{j=1}^{N \mathcal{B}} e^{2 \pi i|P(k, l)|(N \mathcal{B})^{-1} j}\right) e^{2 \pi i|P(k, l)| x(N \mathcal{B})^{-1}}\right\} .
$$


Therefore

$$
|S(T, T+H)| \leq \frac{1}{2} \max _{j}\left\{\sum_{k}\left|\sum_{l}^{*} e^{2 \pi i|P(k, l)| y_{j}}\right|\right\} .
$$

Since $j$ runs over a finite set of positive integers and since $x$ is irrational, $y_{j}$ is irrational and hence $(P(k, l)) y_{j}$ is a quadratic polynomial in $l$ with the leading coefficient irrational. First, we note that $k=0$ trivially gives $o(H)$ to (3.9.13). So, it is enough to consider $k>0$. Let $M=(H / \sqrt{T}) / \sqrt{H / \sqrt{T}}$. For fixed $k>0$, we see that

$$
\sum_{l}^{* *} e^{2 \pi i|P(k, l)| y_{j}}=O(M),
$$

where $* *$ indicates that $l$ belongs to those intervals whose length is $\leq M$. For fixed $k>0$, using Lemma 3.8, we obtain

$$
\sum_{l}^{* * *} e^{2 \pi i|P(k, l)| y_{j}}=o(H / \sqrt{T})
$$

where $* * *$ indicates that $l$ runs over those intervals whose length lies between $M$ and $H / \sqrt{T}$. Therefore from (3.9.13)-(3.9.15), we get

$$
S(T, T+H)=o(H),
$$

since $k \ll \sqrt{T}$.

Case (ii): $\mathbb{K}=\mathbb{Q}(\sqrt{d})$ with $d<0$. When $d=-1$ and -3 , the class number of the field $\mathbb{K}$ is 1 and hence $\zeta_{\mathbb{K}}(s, \mathcal{C})$ will contain a factor $\zeta(s)$. So, for the purpose of our paper, we can assume $d \neq-1$ and $\neq-3$. In this case, the class number of $\mathbb{K}$ is $>1$ and there are two units of $\mathbb{K}$. Hence, we get

$$
P(k, l)=k^{2}+a_{2} k l+b_{2} l^{2} \quad \text { with } 4 b_{2}-a_{2}^{2}>0
$$

and $T(N \mathcal{B}) \leq|P(k, l)| \leq(T+H)(N \mathcal{B})$. Trivially, we get $0 \leq k \ll \sqrt{T}$ and now we can argue as we did in the case (i), and obtain the lemma.

LEMMA 3.10. If $a / \sqrt{\Delta}$ or $c / \sqrt{\Delta}$ is irrational (in particular, if $a, b, c$ are integers and $\Delta$ is not a square) and $H=H(T)$ is such that $H \leq T$ and $H / \sqrt{T}$ tends to infinity with $T$, then

$$
\int_{T}^{T+H} W_{1}(t) d t=o(H)+O\left(T^{1 / 2} \log T\right) .
$$

Proof. Recall $s_{0}=1+i t$. First, we note that from (2.9),

$$
\left(\chi_{1}\left(s_{0}\right)\right)^{-1 / 2}=C_{15} t^{1 / 2}\left(\frac{t \sqrt{\Delta}}{2 \pi e}\right)^{i t}+O\left(t^{-1 / 2}\right),
$$


where $C_{15}=e^{i \pi / 4}(\sqrt{\Delta /(2 \pi)})^{1 / 2}$. Now, we have

$$
\text { (3.10.2) } \begin{aligned}
\int_{T}^{T+H} W_{1}(t) d t & =-i \int_{1 / 2+i T}^{1 / 2+i(T+H)} f_{1}(s) d s \\
& =-i\left\{\int_{1 / 2+i T}^{1+i T}+\int_{1+i T}^{1+i(T+H)}+\int_{1+i(T+H)}^{1 / 2+i(T+H)}\right\} f_{1}(s) d s \\
& =L_{1}+L_{2}+L_{3} \quad \text { (say). }
\end{aligned}
$$

From Lemma 3.5(i) and (2.9), we obtain

$$
L_{1} \ll \int_{1 / 2}^{1} T^{(1-\sigma)+\sigma-1 / 2}(\log T) d \sigma \ll T^{1 / 2} \log T .
$$

Similarly, we obtain

$$
L_{3} \ll T^{1 / 2} \log T .
$$

From Lemma 3.6 and (3.10.1), we have

$$
\begin{aligned}
(3.10 .5) L_{2}= & \int_{T}^{T+H} f_{1}(1+i t) d t \\
= & \int_{T}^{T+H}\left\{C_{15} t^{1 / 2}\left(\frac{t \sqrt{\Delta}}{2 \pi e}\right)^{i t}+O\left(t^{-1 / 2}\right)\right\} \\
& \times\left\{\sum_{\varphi} \frac{e^{-\varphi / X_{3}}}{\varphi^{s_{0}}}+O\left(T^{-3 / 2}(\log T)^{3}\right)\right\} d t \\
= & C_{15} \sum_{\varphi} \frac{e^{-\varphi / X_{3}}}{\varphi} \int_{T}^{T+H} t^{1 / 2}\left(\frac{t \sqrt{\Delta}}{2 \pi e \varphi}\right)^{i t} d t+O\left((\log T)^{5}\right) \\
& +O\left(T^{1 / 2} \sum_{\varphi} \frac{e^{-\varphi / X_{3}}}{\varphi}\right)+O\left(T^{-1}(\log T)^{5}\right) .
\end{aligned}
$$

We have

$$
\begin{aligned}
\sum_{\varphi} \frac{e^{-\varphi / X_{3}}}{\varphi} & \leq \sum_{\varphi \leq X_{3}} \frac{1}{\varphi}+\sum_{\varphi>X_{3}} \frac{e^{-\varphi / X_{3}}}{\varphi} \\
& =\sum_{j \leq X_{3}} \frac{l(j)}{j}+\sum_{j>X_{3}} \frac{l(j) e^{-j / X_{3}}}{j} \\
& \ll \log X_{3}+\int_{1}^{\infty} e^{-v} v^{-1} d v \ll \log T
\end{aligned}
$$


since $X_{3}=\sqrt{\Delta} T^{4}$. Hence, we obtain $(3.10 .7)$

$$
L_{2}=C_{15} \sum_{\varphi} \frac{e^{-\varphi / X_{3}}}{\varphi} \int_{T}^{T+H} t^{1 / 2}\left(\frac{t \sqrt{\Delta}}{2 \pi e \varphi}\right)^{i t} d t+o(H)+O\left(T^{1 / 2} \log T\right) .
$$

To estimate the first term of (3.10.7), we divide the range of $\varphi$ as follows, where $K_{1}=\sqrt{\Delta} /(2 \pi)$ :

$$
\begin{gathered}
{\left[0, K_{1}(\sqrt{T}-1)^{2}\right), \quad\left[K_{1}(\sqrt{T}-1)^{2}, K_{1}(\sqrt{T}+1)^{2}\right),} \\
{\left[K_{1}(\sqrt{T}+1)^{2}, K_{1}(\sqrt{T+H}-1)^{2}\right),} \\
{\left[K_{1}(\sqrt{T+H}-1)^{2}, K_{1}(\sqrt{T+H}+1)^{2}\right),} \\
{\left[K_{1}(\sqrt{T+H}+1)^{2}, X_{3}^{2}\right), \quad\left[X_{3}^{2}, \infty\right) .}
\end{gathered}
$$

Let $\sum_{1}, \sum_{2}, \ldots, \sum_{6}$ be the corresponding parts of the above sum. Now,

$$
\begin{aligned}
\sum_{1} & =C_{15} \sum_{\varphi \leq K_{1}(\sqrt{T}-1)^{2}} \frac{e^{-\varphi / X_{3}}}{\varphi} \int_{T}^{T+H} t^{1 / 2}\left(\frac{K_{1} t}{e \varphi}\right)^{i t} d t \\
& =C_{15} \sum_{r \leq \sqrt{K_{1}}(\sqrt{T}-1)} \sum_{(r-1)^{2} \leq \varphi<r^{2}} \frac{e^{-\varphi / X_{3}}}{\varphi} \int_{T}^{T+H} t^{1 / 2}\left(\frac{K_{1} t}{e \varphi}\right)^{i t} d t \\
& =O\left(\sum_{r \leq \sqrt{K_{1}}(\sqrt{T}-1)} \frac{1}{r} \cdot \frac{T^{1 / 2}}{\left|\log \left(K_{1} T / \varphi\right)\right|}\right) .
\end{aligned}
$$

(We have used (3.1.1) with $\mu=1 / 2$ and the number of terms in the inner sum is $O(r)$.) Since

$$
\left|\log \frac{m}{n}\right| \geq \frac{|m-n|}{m+n}
$$

for any two positive numbers $m, n$, we have

$$
\begin{aligned}
\sum_{1} & =O\left(T^{1 / 2} \sum_{r \leq \sqrt{K_{1}}(\sqrt{T}-1)} \frac{1}{r} \cdot \frac{r+\sqrt{K_{1} T}}{\left|r-\sqrt{K_{1} T}\right|}\right) \\
& =O\left(T^{1 / 2} \sum_{r \leq \sqrt{K_{1}}(\sqrt{T}-1)}\left\{\frac{1}{\left|r-\sqrt{K_{1} T}\right|}+\frac{\sqrt{K_{1} T}}{r\left|r-\sqrt{K_{1} T}\right|}\right\}\right) \\
& =O\left(T^{1 / 2} \log T\right)
\end{aligned}
$$

since

$$
\sum_{r \leq \sqrt{K_{1}}(\sqrt{T}-1)} \frac{1}{\left|r-\sqrt{K_{1} T}\right|}=O(\log T)
$$


and

$$
\begin{aligned}
\sum_{r \leq \sqrt{K_{1}}(\sqrt{T}-1)} \frac{\sqrt{K_{1} T}}{r\left|r-\sqrt{K_{1} T}\right|}=\sum_{\substack{r \leq \sqrt{K_{1} T} / 2 \\
r\left|r-\sqrt{K_{1} T}\right|}} \frac{\sqrt{K_{1} T}}{} & +\sum_{\substack{\sqrt{K_{1} T} / 2<r \leq \sqrt{K_{1}}(\sqrt{T}-1) \\
=}} \frac{\sqrt{K_{1} T}}{r\left|r-\sqrt{K_{1} T}\right|} \\
& O(\log T) .
\end{aligned}
$$

Now, using (3.1.4) with $\mu=1 / 2$, we obtain

$$
\begin{aligned}
\sum_{2} & =\sum_{K_{1}(\sqrt{T}-1)^{2} \leq \varphi \leq K_{1}(\sqrt{T}+1)^{2}} \frac{e^{-\varphi / X_{3}}}{\varphi} \int_{T}^{T+H} t^{1 / 2}\left(\frac{K_{1} t}{e \varphi}\right)^{i t} d t \\
& =O\left(\frac{1}{T} \cdot T\left(K_{1}(\sqrt{T}+1)^{2}-K_{1}(\sqrt{T}-1)^{2}\right)\right)=O\left(T^{1 / 2}\right)
\end{aligned}
$$

and similarly, we get

$$
\sum_{4}=O\left(T^{1 / 2}\right) .
$$

We note that we can use (3.1.2) to estimate $\sum_{5}$ and $\sum_{6}$. Now,

$$
\begin{aligned}
\sum_{5} & =\sum_{K_{1}\left(\sqrt{T+H+1)^{2} \leq \varphi<X_{3}^{2}}\right.} \frac{e^{-\varphi / X_{3}}}{\varphi} \int_{T}^{T+H} t^{1 / 2}\left(\frac{K_{1} t}{e \varphi}\right)^{i t} d t \\
& \ll \sum_{\sqrt{K_{1}}(\sqrt{T+H}+1) \leq r<X_{3}(r-1)^{2} \leq \varphi<r^{2}} \frac{1}{\varphi} \cdot \frac{T^{1 / 2}}{\log \left(\frac{\varphi}{K_{1}(T+H)}\right)} \\
& \ll T^{1 / 2} \sum_{\sqrt{K_{1}}(\sqrt{T+H}+1) \leq r<X_{3}} \frac{1}{r} \cdot \frac{1}{\log \left(\frac{r^{2}}{K_{1}(T+H)}\right)} \\
& \ll T^{1 / 2} \log T .
\end{aligned}
$$

Now,

$$
\begin{aligned}
\sum_{6} & \ll \sum_{\varphi \geq X_{3}^{2}} \frac{e^{-\varphi / X_{3}}}{\varphi} \cdot \frac{T^{1 / 2}}{\log \left(\frac{\varphi}{K_{1}(T+H)}\right)} \\
& \ll T^{1 / 2} e^{-X_{3} / 2} X_{3} \frac{1}{X_{3}^{2}} \ll T^{-10} .
\end{aligned}
$$

It remains only to estimate $\sum_{3}$. Now,

$$
(3.10 .14) \sum_{3}=C_{15} \sum_{K_{1}(\sqrt{T}+1)^{2} \leq \varphi<K_{1}(\sqrt{T+H}-1)^{2}} \frac{e^{-\varphi / X_{3}}}{\varphi} \int_{T}^{T+H} t^{1 / 2}\left(\frac{K_{1} t}{e \varphi}\right)^{i t} d t .
$$


Note that $K_{1}=\sqrt{\Delta} /(2 \pi)$. With $\varphi$ in the range as in (3.10.14), using (3.1.3) with $\mu=1 / 2$ and $\xi=\varphi / K_{1}$, we obtain

$$
\begin{aligned}
\sum_{3}= & \sqrt{2 \pi} C_{15} e^{i \pi / 4} K_{1}^{-1} \sum_{\varphi} e^{-\varphi / X_{3}} e^{-2 \pi i \varphi / \sqrt{\Delta}} \\
& +O\left(T^{9 / 10} \sum_{\varphi} \frac{e^{-\varphi / X_{3}}}{\varphi}\right)+O\left(T^{1 / 2} \sum_{\varphi} \frac{e^{-\varphi / X_{3}}}{\varphi \log \left(\frac{\varphi}{K_{1} T}\right)}\right) \\
& +O\left(T^{1 / 2} \sum_{\varphi} \frac{e^{-\varphi / X_{3}}}{\varphi \log \left(\frac{K_{1}(T+H)}{\varphi}\right)}\right) \\
= & L_{4}+L_{5}+L_{6}+L_{7}, \quad \text { say. }
\end{aligned}
$$

Since $K_{1}(\sqrt{T}+1)^{2} \leq \varphi<K_{1}(\sqrt{T+H}-1)^{2}$ and $X_{3}=K_{1}(\sqrt{T}-1)^{8}$, we note that

$$
e^{-\varphi / X_{3}}=1+O\left(\varphi / X_{3}\right)=1+O\left(T^{-2}\right),
$$

and we use (3.10.7). Also, note that the number of integers in $\left[K_{1}(\sqrt{T}+1)^{2}\right.$, $\left.K_{1}(\sqrt{T+H}-1)^{2}\right)$ is

$$
C_{16} K_{1} H+O(\sqrt{T})=O(H) .
$$

From (3.10.17), we get

$$
\begin{aligned}
L_{5} & \ll T^{9 / 10} \sum_{K_{1}(\sqrt{T}+1)^{2} \leq j<K_{1}(\sqrt{T+H}-1)^{2}} \frac{l(j)}{j} e^{-j / X_{3}} \\
& \ll T^{9 / 10-1} H=o(H) .
\end{aligned}
$$

Using (3.10.7), we obtain,

$$
\begin{aligned}
L_{6} & \ll T^{1 / 2} \sum_{K_{1}(\sqrt{T}+1)^{2} \leq j<K_{1}(\sqrt{T+H}-1)^{2}} \frac{l(j)}{j} e^{-j / X_{3}} \frac{j+K_{1} T}{\left|j-K_{1} T\right|} \\
& \ll T^{1 / 2} \sum_{K_{1}(\sqrt{T}+1)^{2} \leq j<K_{1}(\sqrt{T+H}-1)^{2}} \frac{l(j)}{\left|j-K_{1} T\right|} \\
& \ll T^{1 / 2} \log T
\end{aligned}
$$

and

$(3.10 .20) \quad L_{7} \ll T^{1 / 2}$

$$
\times \sum_{K_{1}(\sqrt{T}+1)^{2} \leq j<K_{1}(\sqrt{T+H}-1)^{2}} \frac{l(j)}{j} e^{-j / X_{3}} \frac{j+K_{1}(T+H)}{\left|j-K_{1}(T+H)\right|}
$$




$$
\begin{aligned}
& \ll T^{1 / 2} \sum_{K_{1}(\sqrt{T}+1)^{2} \leq j<K_{1}(\sqrt{T+H}-1)^{2}} \frac{l(j)}{\left|j-K_{1}(T+H)\right|} \\
& +\frac{T^{1 / 2} K_{1}(T+H)}{K_{1} T} \sum_{\cdots} \frac{l(j)}{\left|j-K_{1}(T+H)\right|} \\
& \ll T^{1 / 2} \log T .
\end{aligned}
$$

Now,

$$
\begin{aligned}
L_{4}= & \sqrt{2 \pi} C_{15} e^{i \pi / 4} K_{1}^{-1} \sum_{K_{1}(\sqrt{T}+1)^{2} \leq \varphi<K_{1}(\sqrt{T+H}-1)^{2}} e^{-\varphi / X_{3}} e^{-2 \pi i \varphi / \sqrt{\Delta}} \\
= & \sqrt{2 \pi} C_{15} e^{i \pi / 4} K_{1}^{-1} \sum_{\ldots} e^{-2 \pi i \varphi / \sqrt{\Delta}}+O\left(\sum_{\ldots} \varphi / X_{3}\right) \\
= & \sqrt{2 \pi} C_{15} e^{i \pi / 4} K_{1}^{-1} \sum_{K_{1}(\sqrt{T}+1)^{2} \leq \varphi<K_{1}(\sqrt{T+H}-1)^{2}} e^{-2 \pi i \varphi / \Delta} \\
& +O\left(T^{1+\varepsilon} H / X_{3}\right) \\
= & \sqrt{2 \pi} C_{15} e^{i \pi / 4} K_{1}^{-1} \\
& \times \sum_{K_{1}(\sqrt{T}+1)^{2} \leq \varphi<K_{1}(\sqrt{T+H}-1)^{2}} e^{-2 \pi i \varphi / \sqrt{\Delta}}+o(1)
\end{aligned}
$$

since $X_{3} \gg T^{4}$. Now, suppose that $c / \sqrt{\Delta}$ is irrational. Then

$$
\begin{aligned}
\left|\sum e^{-2 \pi i \varphi / \sqrt{\Delta}}\right| & =\left|\sum_{m} \sum_{n} e^{-2 \pi i\left(a m^{2}+b m n+c n^{2}\right) / \sqrt{\Delta}}\right| \\
& \leq \sum_{m}\left|\sum_{n} e^{-2 \pi i\left(a m^{2}+b m n+c n^{2}\right) / \sqrt{\Delta}}\right| .
\end{aligned}
$$

Since $H / \sqrt{T}$ tends to infinity with $T$, the range of values of $n$ consists of one or two intervals, the length of each of which tends to infinity. Hence by Lemma 3.8, we get

$$
\sum_{n} e^{-2 \pi i\left(a m^{2}+b m n+c n^{2}\right) / \sqrt{\Delta}}=o\left(\sum_{n} 1\right)
$$

and therefore from Lemma 3.2 we obtain

$$
\begin{aligned}
& \sum_{m} \sum_{n} e^{-2 \pi i\left(a m^{2}+b m n+c n^{2}\right) / \sqrt{\Delta}} \\
& \quad=o\left(\sum_{m} \sum_{n} 1\right)=o(R(T+H)-R(T))=o(H)+o(\sqrt{T}) .
\end{aligned}
$$

If $a / \sqrt{\Delta}$ is irrational, a similar argument holds with $m$ and $n$ interchanged. This proves the lemma. 
Lemma 3.11. Let $H=H(T)$ be such that $H \leq T$ and $H / \sqrt{T}$ tends to infinity with $T$. If $\lambda$ is not a perfect square, then

$$
\begin{aligned}
\text { (i) } \int_{T}^{T+H} W_{2}(t) d t & =o(H)+O\left(T^{1 / 2} \log T\right) \text {, } \\
\text { (ii) } \int_{T}^{T+H} W_{3}(t) d t & =o(H)+O\left(T^{1 / 2} \log T\right) .
\end{aligned}
$$

Proof. We note that $\lambda=|d|$ is not a perfect square so that $\sqrt{\lambda}$ is irrational. Again, we notice that from (2.9),

$$
\left(\chi_{j}\left(s_{0}\right)\right)^{-1 / 2}=C_{16} t^{1 / 2}\left(\frac{t \sqrt{\lambda}}{2 \pi e}\right)^{i t}+O\left(t^{-1 / 2}\right) \quad \text { for } j=2,3,
$$

where $C_{16}=e^{i \pi / 4}(\sqrt{\lambda} /(2 \pi))^{1 / 2}$. Instead of $K_{1}$ in the proof of Lemma 3.10, we take $K_{2}=\sqrt{\lambda} /(2 \pi)$. Now, the proof for (i) and (ii) is the same as for Lemma 3.10.

Lemma 3.12 (see Theorem 1 of [10]). Let $C_{17} \geq 1$ and $1=\lambda_{1}<\lambda_{2}<\ldots$ be such that $1 / C_{17} \leq \lambda_{n+1}-\lambda_{n} \leq C_{17}$. Let $1=a_{1}, a_{2}, \ldots$ be a sequence of complex numbers with $\left|a_{n}\right| \leq(n H)^{C_{17}}$ where $(\log T)^{10} \leq H \leq T$. Suppose $F(s)=\sum_{n=1}^{\infty} a_{n} \lambda_{n}^{-s}$ is analytically continuable in an infinite system of rectangles defined by $\{\sigma \geq 1 / 2, T \leq t \leq T+H\}$ and there $\max |F(s)|<T^{C_{17}}$. Then

$$
\int_{T}^{T+H}|F(1 / 2+i t)| d t \gg H,
$$

where the implied constant is effective.

Proof. First, we choose $C_{18}$ large enough such that

$$
1 \ll F(\sigma+i t) \ll 1 \quad \text { for } \sigma \geq 1 / 2+C_{18} .
$$

Consider the rectangle $R_{1}$ defined by the line segments joining the points $1 / 2+i T, 1 / 2+2 C_{18}+i T, 1 / 2+2 C_{18}+i(T+H), 1 / 2+i(T+H)$ and $1 / 2+i T$. Let $s_{1}=1 / 2+C_{18}+i t_{1}$, where $T+H / 10 \leq t_{1} \leq T+H-H / 10$. By the residue theorem, for $X>0$ we have

$$
\begin{aligned}
F\left(s_{1}\right) & =\frac{1}{2 \pi i} \int_{R_{1}} \frac{F(s) X^{s-s_{1}} e^{\left(s-s_{1}\right)^{2}}}{s-s_{1}} d s \\
& =H_{11}+H_{12}+V_{11}+V_{12} \quad \text { (say) }
\end{aligned}
$$

where $H_{11}, H_{12}$ are the horizontal lines contributions and $V_{11}, V_{12}$ are the 
vertical lines contributions. We note that

$$
\begin{aligned}
H_{11} & \ll \int_{1 / 2}^{1 / 2+2 C_{18}} i \frac{|F(\sigma+i T)| X^{\sigma-1 / 2-C_{18}} e^{\left(\sigma-1 / 2-C_{18}\right)^{2}-\left(T-t_{1}\right)^{2}}}{\left|\sigma-1 / 2-C_{18}+i\left(T-t_{1}\right)\right|} d \sigma \\
& \ll T^{C_{17}}\left(X^{C_{18}}+X^{-C_{18}}\right) e^{-C_{19} H^{2}}
\end{aligned}
$$

and similarly,

$$
H_{12} \ll T^{C_{17}}\left(X^{C_{18}}+X^{-C_{18}}\right) e^{-C_{19} H^{2}} .
$$

Now, from (3.12.1),

$$
\begin{aligned}
V_{12} & =\frac{1}{2 \pi} \int_{T}^{T+H} \frac{F\left(1 / 2+2 C_{18}+i t\right) X^{C_{18}+i\left(t-t_{1}\right)} e^{C_{18}^{2}-\left(t-t_{1}\right)^{2}+i()}}{C_{18}+i\left(t-t_{1}\right)} d t \\
& \ll X^{C_{18}} \int_{T}^{T+H} e^{-\left(t-t_{1}\right)^{2}} d t .
\end{aligned}
$$

Also, we have

$$
\begin{aligned}
V_{11} & =\frac{1}{2 \pi} \int_{T}^{T+H} \frac{F(1 / 2+i t) X^{-C_{18}+i\left(t-t_{1}\right)} e^{C_{18}^{2}-\left(t-t_{1}\right)^{2}+i(~)}}{-C_{18}+i\left(t-t_{1}\right)} d t \\
& \ll X^{-C_{18}} \int_{T}^{T+H} \frac{|F(1 / 2+i t)| e^{-\left(t-t_{1}\right)^{2}}}{\left|-C_{18}+i\left(t-t_{1}\right)\right|} d t .
\end{aligned}
$$

From (3.12.1)-(3.12.6), we obtain,

$$
\begin{aligned}
H & \ll \int_{T+H / 10}^{T+H-H / 10}\left|F\left(s_{1}\right)\right| d t_{1} \\
& \ll X^{C_{18}} H+X^{-C_{18}} \int_{T+H / 10}^{T+9 H / 10} d t_{1} \int_{T}^{T+H} \frac{|F(1 / 2+i t)| e^{-\left(t-t_{1}\right)^{2}}}{\left|-C_{18}+i\left(t-t_{1}\right)\right|} d t \\
& \ll X^{C_{18}} H+X^{-C_{18}}\left(\int_{T}^{T+H}|F(1 / 2+i t)| d t\right) .
\end{aligned}
$$

We choose $X$ such that

$$
X^{C_{18}} H=X^{-C_{18}}\left(\sqrt{H}+\int_{T}^{T+H}|F(1 / 2+i t)| d t\right) .
$$

From (3.12.8), it clearly follows that $1 / T^{C_{19}} \ll X \ll T^{C_{20}}$. First choose 
$X=H^{-\varepsilon}$ to get

$$
\int_{T}^{T+H}|F(1 / 2+i t)| d t \gg H^{1-C_{18} \varepsilon}
$$

then choose

$$
X=H^{-1}\left(\int_{T}^{T+H}|F(1 / 2+i t)| d t\right)^{1 / 2 C_{18}}
$$

to get

$$
\int_{T}^{T+H}|F(1 / 2+i t)| d t \gg H
$$

which proves the lemma.

Remark. For a more precise and general version of Lemma 3.12, we refer to $[2]$.

4. Proof of the inequality (1.3). It is enough to prove (1.3) in the case of $Z(s)$. Others follow in the similar way. If $W_{1}(t)$ is of constant sign over the interval $[T, T+H]$, then we have

$$
\left|\int_{T}^{T+H} W_{1}(t) d t\right|=\int_{T}^{T+H}\left|W_{1}(t)\right| d t .
$$

By Lemmas 3.10 and 3.12, it follows that

$$
o(H)+O\left(T^{1 / 2} \log T\right)=\left|\int_{T}^{T+H} W_{1}(t) d t\right|=\int_{T}^{T+H}\left|W_{1}(t)\right| d t>C_{21} H .
$$

Since all the constants are effective, if we choose $H=C_{22} T^{1 / 2} \log T$ where $C_{22}$ is a large effective positive constant, the inequality (4.2) is contradicted and hence $W_{1}(t)$ has a zero in every interval $\left(T, T+C_{22} T^{1 / 2} \log T\right)$ with $T$ large enough. Now (1.3) follows.

Acknowledgements. The author wishes to thank Professor K. Ramachandra for fruitful discussions and for his constant encouragement, particularly for a simple proof of Lemma 3.12. The author is very much indebted to him for checking the manuscript thoroughly. The author wishes to thank the referee for pointing out some misprints and some simplification of Lemma 3.12 in the earlier version of this manuscript.

\section{References}

[1] R. Balasubramanian, An improvement of a theorem of Titchmarsh on the mean square of $|\zeta(1 / 2+i t)|$, Proc. London Math. Soc. (3) 36 (1978), 540-576. 
[2] R. Balasubramanian and K. Ramachandra, Proof of some conjectures on the mean-value of Titchmarsh series. I, Hardy-Ramanujan J. 13 (1990), 1-20.

[3] B. C. Berndt, The number of zeros of the Dedekind zeta-function on the critical line, J. Number Theory 3 (1971), 1-6.

[4] K. Chandrasekharan and R. Narasimhan, Zeta-functions of ideal classes in quadratic fields and their zeros on the critical line, Comment. Math. Helv. 43 (1968), $18-30$.

[5] E. Hecke, Über Dirichlet-Reihen mit Funktionalgleichung und ihre Nullstellen auf der Mittelgeraden, München Akad. Sitzungsber. II, 8 (1937), 73-95.

[6] -, Über die Bestimmung Dirichletscher Reihen durch ihre Funktionalgleichung, Math. Ann. 112 (1936), 664-699.

[7] E. Landau, Vorlesungen über Zahlentheorie, VII. Teil, Kap. 7, Chelsea, 1947.

[8] H. S. A. Potter, Approximate equations for the Epstein zeta-function, Proc. London Math. Soc. (2) 36 (1934), 501-515.

[9] H. S. A. Potter and E. C. Titchmarsh, The zeros of Epstein's zeta-functions, Proc. London Math. Soc. 39 (1935), 372-384.

[10] K. Ramachandra, Progress towards a conjecture on the mean-value of Titchmarsh series, in: Recent Progress in Analytic Number Theory, H. Halberstam and C. Hooley (eds.), Vol. 1, Academic Press, 1981, 303-318.

[11] H. M. Stark, L-functions and character sums for quadratic forms (I), Acta Arith. 14 (1968), 35-50.

[12] E. C. Titchmarsh, The Theory of the Riemann Zeta-Function, 2nd ed., revised by D. R. Heath-Brown, Oxford Sci. Publ., Clarendon Press, Oxford, 1986.

SCHOOL OF MATHEMATICS

TATA INSTITUTE OF FUNDAMENTAL RESEARCH

HOMI BHABHA ROAD

BOMBAY 400 005, INDIA 